\title{
Introduction to the 2005 long course
}

\author{
Bruce R Smoller \\ Department of Pathology, University of Arkansas for Medical Sciences, Little Rock, AR, USA
}

\begin{abstract}
Dermatopathology, as with other fields of pathology, has undergone major changes in the last 25 years. Our understanding of epidermally derived neoplasms becomes far more advanced with each passing year. Evidence for this progress is readily apparent upon reviewing textbooks of dermatopathology over time and in examining the course contents of the long-course presentations at the United States-Canadian Academy of Pathology annual meetings. What was once a simple discussion of melanocytic neoplasms (thought to be a variant of sarcoma) has become a reasonably complex analysis of the histologic, immunologic, and molecular aspects of melanocytic neoplasia. Similarly, our understanding of the development of cutaneous squamous cell carcinoma is greatly enhanced. The understanding of the molecular alterations that result in full transformation offers great insight into carcinogenesis. This introductory essay briefly traces this progression in setting the stage for the series of more detailed chapters about epidermal neoplasms that follow.

Modern Pathology (2006) 19, S1-S3. doi:10.1038/modpathol.3800507
\end{abstract}

Keywords: dermatopathology; epidermis; neoplasms

Dermatopathology has evolved rapidly over the past several decades, in much the same manner as other fields in pathology and medicine overall. As I have said around our multiheaded microscope many times, it seems like half of what we discuss during our sign-out sessions did not even exist during my residency (in the mid-1980s). Our field is forever advancing and changing. It is sometimes instructive to take a look backwards, briefly, to see where we have been, before presenting a seminar concerning the current state of the discipline and speculating on future developments.

The papers that follow will concentrate on epidermal neoplasms. Over the past 40 years, there have been two prior Long Courses at the United States-Canadian Academy of Pathology dedicated to dermatopathology. A study of the syllabi from these two seminars serves to demonstrate how our subspecialty has advanced. In 1968, Dr Elston Helwig directed a half-day seminar entitled 'Geographic Pathology of the Skin'. There were four talks, including one dedicated to melanocytic tumors, another to mast cells, and the other two devoted to skin diseases in Africa and Vietnam. These topics are obviously quite broad, and today each one could easily serve as the topic for a series

Correspondence: Dr BR Smoller, Department of Pathology, University of Arkansas for Medical Sciences, 4300 W. Markham, Little Rock, AR 72205, USA.

E-mail: smollerbrucer@uams.edu

Received 13 September 2005; accepted 28 September 2005 of lectures or a full-day seminar. As we prepared for the current course, it was unthinkable to try to cover more than tumors of the epidermis, and serious thought was given to limiting the topic even farther.

In 1987, Dr Martin Mihm Jr directed a long course on Melanocytic Neoplasms. Certainly, much more was known, in 1987, about the biology of and pathologic changes seen in melanocytic tumors than was the case 20 years prior. However, most of these lectures were based upon the analysis of routine histologic sections, with some attention paid towards immunostaining as it was becoming incorporated into diagnostic dermatopathology. Molecular diagnostic, karyotyping and consideration of genetic aberrations did not figure into the pathologists' concerns about the pathogenesis of melanoma.

As one studies the subsequent chapters, it will become apparent that while routine histologic examination still retains its central position in dermatopathology, ancillary techniques are increasingly crucial in our attempt to make earlier diagnoses and to provide additional prognostic information for our contributing clinicians and their patients. The first several chapters deal with some of the benign melanocytic neoplasms that provide us with the most difficulty in diagnosis and whose biologic behavior is not fully understood. Our current lack of consensus regarding the diagnosis and significance of these entities reflects the present state of uncertainty and ignorance on our parts. However, it also reflects our ever-improving ability to discern minute differences and to use these differences to identify neoplasms that may foretell 
different clinical courses. Certainly, it represents progress to have identified clinicopathologic entities whose meaning remains unknown, all the while allowing scientific discovery to further elucidate their significance.

We have made enormous strides in understanding the biologic behavior of melanocytic neoplasms. In 1911, Schamberg wrote 'Primary melanotic sarcoma or melanosarcoma is one of the most common and most malignant forms of the disease [sarcoma]. It usually has its origin in an irritated pigmented nevus, although other pigmented patches may be the site of development.' ${ }^{1}$ Certainly, there is some truth to this statement, although I think no longer would we classify melanoma as a sarcoma or consider it to be one of the most malignant forms of sarcoma. One might also take exception with the assumption that most melanomas arise from irritated pigmented nevi.

Dr Shamberg proceeds to state, 'We are in complete darkness as to the cause of sarcoma. It occurs at all ages, and is at times congenital.' Again, much truth exists in this statement. As will be addressed to some degree in this symposium, we have made some progress in our understanding of the pathogenesis of melanoma. However, there is still much about which we have little understanding. He finishes his discussion with, 'The prognosis is always grave, most cases terminating fatally.' As will be evident from the subsequent discussions, this final assessment has been markedly altered. We have made significant progress in our abilities to diagnose progressively earlier lesions, often before there is even metastatic capability. While we have made some strides in our ability as physicians to treat melanoma, unfortunately, these efforts have not yet borne tremendous success, further emphasizing the importance of our abilities to detect accurately the earliest possible lesions in the evolution of this neoplasm.

In the second edition of Dr Lever's classic textbook, it is curious to note that under the section entitled, 'Melanomas', there are subsections including pigmented nevus, blue nevus and lentigo. ${ }^{2}$ Further, the entire chapter is about 15 pages in length. Perhaps, there had been some progress from 1911 to 1954, the time of this publication, but clearly, our understanding of pigmented lesions was relatively primitive at this time. If one compares Lever's volume with the presentation of 'Lentigines, Nevi and Melanomas' that spans about 40 pages in the latest edition of Dr Weedon's textbook, the differences are manifest. ${ }^{3}$ As exhaustive as this latter volume appears, it concentrates almost exclusively on diagnostic criteria and clinicopathologic correlation, with relatively little space allocated for developments in our understanding of the pathobiology of these lesions. Clearly, there has been an explosion of information about melanocytic tumors, as will be evident from the first portion of this symposium. We have truly come a long way (and as will be addressed in the epilogue, there is a long way still to go).

A similar story can be told about keratinocytic neoplasms. In his textbook from early 20th century, Dr Shamburg states, 'The essential process in epithelioma is the proliferation of epithelial cells and their extension into structures not normally the seat of the cells. Epithelioma cutis must have its origin in the epithelium of the epidermis or in the epithelial lining of the glandular structures in the skin. ${ }^{1}$ Note that there is no attempt in this statement (or within the textbook as a whole) to subdivide these tumors into squamous cell carcinomas, basal cell carcinomas or the myriad appendageal neoplasms that we have come to recognize. Dr Shamburg continues with, 'Accumulated experience points strongly toward continuous or frequently repeated irritation as the most important factor in the production of epithelioma', and adds, 'Chemic rays of light are certainly a factor in the production of many skin cancers, particularly those that begin as keratoses.' It is remarkable how these observations have persisted over nearly a century. However, as will be described in subsequent chapters, the mechanisms by which this transformation occurs have been much more fully elucidated. The observation has now been distilled down to a discussion of DNA mutations.

By 1927, Gilchrist attempted a subclassification system with, 'Epithelioma: Term applied to skin cancers, of which there are two main types: A. Squamous cell, B. Basal cell or rodent-most frequently in middle or old age-usually single lesion-begins in scaly spot, excoriation, fissure, nodule, wart or mole-frequently result of chronic irritation'. ${ }^{4}$ At this time, it was apparently understood that keratinocytic neoplasms were not a homogeneous group of neoplasms. However, while the clinical description of these tumors is still valid, the putative precursor lesions may not be entirely accurate as per our current understanding. It is interesting, although, to note the role attributed to chronic irritation persisted in the literature and probably still remains valid today. Again, the simple observation has led to a line of investigation that has borne great fruit in our understanding of the etiology of carcinoma.

By 1949, Dr Lever had divided keratinocytic neoplasms into squamous cell carcinoma and Bowen's disease (to which he dedicated 10 pages) and a separate section on cutaneous appendage tumors. $^{2}$ In their 1972 textbook entitled, Dermal Pathology, Graham and Helwig provided a greatly expanded chapter on squamous cell carcinoma, subdividing the tumor into various subtypes based solely upon morphologic criteria. ${ }^{5}$ Here, we are introduced to the keratoacanthoma, adenoid and spindle cell types of squamous cell carcinoma. The descriptions grew more elaborate, as did the classification system, but there was still no real attention paid to pathogenesis or prognostic indicators. Later, 
Dr Weedon again provided a remarkably thorough review in his chapter entitled, 'Tumors of the Epidermis', wherein he clearly delineates the distinctions between squamous cell carcinomas, basal cell carcinomas and appendageal tumors. ${ }^{3}$ There is extensive description of discriminating histologic features, although not an extensive review of pathobiology (which falls outside the purview of the volume). Nonetheless, this chapter spans 50 pages and is remarkably more elaborate than the similar sections of earlier textbooks of dermatopathology.

The latter chapters in this symposium deal with aspects of tumors derived from keratinocytes. As is the case with the melanocytic neoplasms, there is no attempt to address every single tumor derived from this cell type, but rather to concentrate on some of the diagnostic difficulties and the new developments in our understanding of the pathobiology of this wide spectrum of neoplasms.

In conclusion, it is our hope that this symposium will serve as a readily accessible update on the current thinking regarding the diagnosis and patho- genesis of a sampling of epidermally derived neoplasms. I think that it will become evident just how far we have come in our ability to understand what causes these tumors, how to diagnose them, and what features may be helpful in determining ultimate prognosis. Much still remains to be discovered in terms of their prevention and treatment, and the underlying science likely provides the key to solving these problems.

\section{References}

1 Schamberg JF. Diseases of the Skin, 2nd edn. WB Saunders: Philaelphia, PA, 1911.

2 Lever WF. Histopathology of the Skin. JB Lippincott Company: Philadelphia, PA, 1954.

3 Weedon D. Skin Pathology, 2nd edn. Churchill Livingstone: London, England, 2002.

4 Gilchrist TC. Outlines of Common Skin Diseases. The Williams and Wilkins Company: Baltimore, 1927.

5 Graham JH, Johnson WC, Helwig EB (eds). Dermal Pathology. Harper \& Row: Hagerstown, MD, 1972. 